\title{
Colonic stenting for malignant large bowel obstruction is safe and effective: a single-surgeon experience
}

\author{
Man Hon $\underline{\text { Tang}}{ }^{1}$, MBChB, MRCs, Talisa $\underline{R o s s}^{2}$, MBChB, Shen Ann $\underline{Y e o}^{1}$, MBBS, FRCs, Chee Yung $\underline{\operatorname{Ng}}{ }^{1}$, MBBS, FRCS
}

INTRODUCTION Self-expanding metal stents (SEMS) can be used as a bridge to surgery (BTS) or for palliation in the treatment of malignant large bowel obstruction. This case series evaluates the short-term outcomes of SEMS and success rates over time.

METHODS A total of 75 consecutive patients who underwent colonic stenting for malignant colonic obstruction over a period of six years were included. This time period was subdivided into two equal parts for analysis. The procedure was carried out by a single surgeon from a tertiary institution in Singapore.

RESULTS Technical success was reported in $93.3 \%$ of cases and clinical success in $81.3 \%$ of cases, with better success rates in the second half of the study $(89.2 \%$ vs. $73.7 \% ; p<0.05)$. There were seven cases of inadequate decompression and two cases of colonic perforation. The median duration from stent insertion to surgery was ten days, and the median postoperative length of stay was six days.

CONCLUSION SEMS are a safe and effective way of relieving malignant large bowel obstruction, including those that are proximal. The improvement in success over time reflects the importance of having an experienced endoscopist carry out the procedure to ensure optimum success rates.

Keywords: colorectal neoplasms, colorectal surgery, intestinal obstruction, self-expandable metallic stents

\section{INTRODUCTION}

Colorectal cancer is the third most common cancer in the world, with up to 1.4 million new cases diagnosed each year. ${ }^{(1)}$ About $7 \%-30 \%$ of colorectal cancers can present acutely with intestinal obstruction, especially if the tumour is located at or distal to the splenic flexure. ${ }^{(2)}$

Emergency surgery is the traditional approach in cases of malignant large bowel obstruction, to defunction or resect the affected bowel and prevent further bowel ischaemia or perforation. The feasibility of primary anastomosis depends on the condition of the colon as well as the patient's haemodynamic status. Stoma creation, either in the form of a diverting colostomy or an end colostomy, can occur at rates of up to $40 \%$ in the emergency setting. ${ }^{(3)}$ An Association of Coloproctology of Great Britain and Ireland audit in 2003, which included more than 8,000 patients, showed that emergency surgery for large bowel obstruction carries a mortality of up to $20 \% .{ }^{(4)}$ In addition, there are also potential psychological consequences for the patient from the formation of a stoma. ${ }^{(5)}$

The introduction of colonic stenting over 20 years ago offered an alternative approach to dealing with malignant large bowel obstructions. ${ }^{(6)}$ It can act as a bridge to surgery (BTS), allowing temporary bowel decompression by restoring luminal patency. Definitive surgical resection can then be performed in a more elective setting and patients' comorbidities can be further optimised. In addition, colonic stenting can also be used in a palliative setting. Three separate meta-analyses comparing emergency surgery and colonic stenting as BTS for obstructed colorectal cancer showed that stenting had higher primary anastomotic rates with lower stoma rates. There was, however, no advantage in terms of overall mortality and long-term survival outcomes. ${ }^{(2,7,8)}$ Furthermore, colonic stenting had technical and clinical success rates of $70.7 \%-94.3 \%$ and $69.0 \%-96.0 \%$, respectively, with higher rates seen in single-operator series compared to data from pooled randomised controlled trials. ${ }^{(8-10)}$ The main adverse complication of colonic stenting is perforation: $6 \%-9 \%$ of stenting cases have clinical evidence of perforation, while up to $14 \%$ of cases have silent perforation. Two randomised controlled trials were stopped prematurely for stent-related complications. ${ }^{(8,11)}$ There is also increasing evidence that stenting should only be considered in a selected group of patients. ${ }^{(12)}$ The experience of the clinician is crucial, both in terms of technicality, as colonic stenting is considered an advanced endoscopic procedure, and clinical judgement in identifying suitable patients for stenting. ${ }^{(12)}$

In this retrospective audit review, we aimed to evaluate a case series of patients who underwent colonic stenting in our institution, performed by a single surgeon over a period of six years, from 2009 (when he first started performing the procedure independently) to 2015. Our goal was to demonstrate that in experienced hands, colonic stenting is safe and effective for malignant large bowel obstruction. Our main outcomes were to review the success rate of stenting, rate of anastomosis and rate of stoma creation for this group of patients. The long-term and survival outcomes will be addressed in subsequent publications.

\section{METHODS}

This was a retrospective case series of all consecutive patients from a prospectively collected electronic database who 
Table I. Patient demographics and surgical information.

\begin{tabular}{|c|c|c|c|c|}
\hline \multirow[t]{2}{*}{ Parameter } & \multicolumn{3}{|c|}{ No. (\%) } & \multirow[t]{2}{*}{ p-value } \\
\hline & Overall $(n=75)$ & $2009-2012(n=38)$ & $2012-2015(n=37)$ & \\
\hline Male & $46(61.3)$ & $22(57.9)$ & $24(64.9)$ & 0.81 \\
\hline Age* & $68.8 \pm 14.7(36-102)$ & $70.3 \pm 15.1(36-102)$ & $67.6 \pm 14.2(37-91)$ & 0.37 \\
\hline \multicolumn{5}{|l|}{ Tumour location } \\
\hline Sigmoid & $38(50.7)$ & $20(52.6)$ & $18(48.6)$ & 0.61 \\
\hline Descending & $18(24.0)$ & $9(23.7)$ & $9(24.3)$ & 0.57 \\
\hline Splenic & $9(12.0)$ & $3(7.9)$ & $6(16.2)$ & 0.48 \\
\hline Transverse & $4(5.3)$ & $1(2.6)$ & $3(8.1)$ & 0.11 \\
\hline Hepatic flexure & $1(1.3)$ & $1(2.6)$ & $0(0)$ & 0.38 \\
\hline Rectosigmoid & $5(6.7)$ & $4(10.5)$ & $1(2.7)$ & 0.20 \\
\hline Stent intent & & & & 0.09 \\
\hline Bridge to surgery & $57(76.0)$ & $25(65.8)$ & $32(86.5)$ & \\
\hline Palliative & $18(24.0)$ & $13(34.2)$ & $5(13.5)$ & \\
\hline \multicolumn{5}{|l|}{ Success rate } \\
\hline Technical & $70(93.3)$ & $35(92.1)$ & $35(94.6)$ & 0.31 \\
\hline Clinical & 61 (81.3) & 28 (73.7) & 33 (89.2) & $<0.05$ \\
\hline
\end{tabular}

*Data presented as mean \pm standard deviation (range).

underwent attempted colonic stenting by a single surgeon for malignant colorectal cancer. The inclusion criteria were: (a) aged over 18 years; and (b) underwent treatment with a metallic stent for malignant large bowel obstruction in any part of the colon. Most patients were admitted as emergency cases with clinical evidence of large bowel obstruction but without clinical and radiological evidence of perforation or bowel infarction. Patients who had a bowel obstruction from a benign cause or an extracolonic malignancy were excluded.

Patient demographics were recorded, including age, gender, past medical history and ASA (American Society of Anesthesiologists) grade. Included disease characteristics were site of tumour, presence of metastases and degree of obstruction. Intervention variables were date of stent, intention (i.e. palliative or BTS), duration from diagnosis to stent insertion, and the size and type of self-expanding metal stent (SEMS) deployed. Patients classified as having an impending obstruction were those who had a very narrow lumen on endoscopy, to the extent that the scope could not be passed through. Although these patients did not have any clinical or radiological evidence of obstruction, they usually had non-resectable metastatic disease, and therefore, the objective of stenting was palliation. For patients with subsequent surgical resection, details of surgery such as anastomosis, stoma creation, postoperative complications and length of hospital stay were also recorded. The cases were subdivided into two equal time periods, 2009-2012 and 2012-2015, for the purpose of comparison to assess if there was an improvement in success rates over time. A p-value $<0.05$ was considered statistically significant.

The primary outcome was the number of clinical and technical successes after the placement of a SEMS. Technical success was defined as the successful placement of the stent in situ across the length of the obstruction. Clinical success was defined as the absence of complications, with clinical and radiological evidence of bowel decompression.
All colonic stents were performed in the institution's dedicated endoscopy suite under sedation. A guidewire was first passed through the stenotic tumour under fluoroscopic guidance before deploying the SEMS. After the procedure, all patients were monitored clinically and radiologically for evidence of bowel decompression to determine clinical success. Final histopathological reports were also examined post resection to determine cancer stage and evidence of microscopic perforations.

\section{RESULTS}

Colonic SEMS insertion was attempted on a total of 75 patients during the study period (Table I). Their mean age was 68.8 (range $36-102)$ years and the majority were male $(61.3 \%)$. The vast majority of the tumours stented were distal tumours, and half of them $(50.7 \%)$ were located in the sigmoid colon. Stenting was a BTS for $57(76.0 \%)$ patients and for palliation in the remainder $(24.0 \%, \mathrm{n}=18)$. The majority $(\mathrm{n}=69)$ of stented patients presented with acute intestinal obstruction and stenting was performed within a median time of $15 \pm 12.5$ (range 2-60) hours from diagnosis. A small number of patients $(n=6)$ had stenting for impending tumour obstruction despite not exhibiting any clinical symptoms of obstruction. For these patients, the median time lapse to stenting was $3 \pm 12.4$ (2-30) days from diagnosis.

The length of the stent used in each case was decided based on the length of the obstructive lesion seen on computed tomography. The majority of the stents were the WallFlex ${ }^{\mathrm{TM}}$ colonic stent (Boston Scientific, Marlborough, MA, USA). Three patients had insertion of the Niti-S ${ }^{\text {TM }}$ enteral colonic stent (Taewoong Medical Co Ltd, Gimpo-si, Gyeonggi-do, South Korea) (Table II).

The overall technical success rate was $93.3 \%$; in four cases, the guidewire could not be passed through safely, and in one case, the stent migrated proximally immediately after it was deployed. The overall clinical success rate was $81.3 \%$, with 
better success rates in the later cohort in $2012-2015$ (89.2\% vs. $73.7 \% ; p<0.05)$. The causes of clinical failure were inadequate decompression $(n=7)$ and colonic perforation $(n=2)$. For the two cases of perforation from stent insertion, both patients underwent emergency laparotomy and Hartmann's procedure. Fig. 1 depicts the radiological findings in some of the unsuccessful cases.

For the 57 patients who had surgery, the median duration from stenting to surgical resection was 10 (range 0-21) days (Table III). $63.2 \%$ of the surgical resections were performed as open procedures, although there was a trend towards laparoscopic surgeries in the second half of the study period. All patients with successful stenting underwent subsequent surgery with primary anastomosis without stoma, except one patient who had an elective Hartmann's procedure due to his age and comorbidities. Emergency surgeries were performed on all patients who had unsuccessful stent insertion; two patients had a Hartmann's procedure and one had colonic resection

Table II. Details of the self-expanding metal stents used.

\begin{tabular}{|c|c|}
\hline Stent type & No. \\
\hline \multicolumn{2}{|c|}{ WallFlex $^{\mathrm{Tm}}(\mathrm{mm})$} \\
\hline $22 \times 60$ & 10 \\
\hline $22 \times 90$ & 8 \\
\hline $25 \times 60$ & 22 \\
\hline $25 \times 90$ & 33 \\
\hline $25 \times 120$ & 1 \\
\hline \multicolumn{2}{|c|}{ Niti-S ${ }^{\mathrm{TM}}(\mathbf{m m})$} \\
\hline $24 \times 80$ & 2 \\
\hline $24 \times 100$ & 3 \\
\hline
\end{tabular}

Total no. of stents does not add up to 75 , as there were some cases in which the stent was unable to be deployed and some patients needed more than 1 stent. with a temporary ileostomy, while the remaining 11 patients had primary anastomosis without stoma creation. Our intentionto-treat analysis showed that $93.0 \%$ of patients had primary anastomosis after colonic resection and only $10.5 \%$ required stoma creation. The anastomotic rate was higher for patients with successful stenting, although the difference was not statistically significant $(95.3 \%$ vs. $85.7 \% ; p=0.22)$. The stoma creation rate was significantly lower in patients who were successfully stented (4.7\% vs. 28.6\%; $p=0.03$ ) (Table III).

Among the 18 patients who received palliative stenting, there was only one case of unsuccessful stenting, which eventually required a colostomy. There were two cases of subsequent stent blockage, one at four months and the other at two years after initial stent placement. The remaining patients did not require further intervention after initial colonic stenting.

The final tumour grading from histopathological examination is shown in Table IV. As expected, there was a higher percentage of more advanced disease among obstructed tumours. No microscopic perforations were found on pathological examination. The median length of hospital stay was four days after colonic stenting, and six days after interval surgical resection of colorectal cancer.

A cumulative sum (CUSUM) chart was plotted to show the trend in clinical success over time (Fig. 2). As shown by the upward slope of the graph, there were increasing rates of clinical success after 35 cases.

\section{DISCUSSION}

Since colonic SEMS was introduced in the 1990s, many studies have examined its role in the treatment of malignant colonic obstruction. Watt et $\mathrm{al}^{(13)}$ showed that stenting is less risky than

Table III. Surgical details of patients who underwent surgical resection after stenting.

\begin{tabular}{|c|c|c|c|}
\hline \multirow[t]{2}{*}{ Parameter } & \multicolumn{3}{|c|}{ No. (\%) } \\
\hline & Overall $(n=57)$ & $2009-2012(n=25)$ & $2012-2015(n=32)$ \\
\hline Duration till surgery* (day) & $10 \pm 6(0-21)$ & $11 \pm 6(0-21)$ & $10 \pm 5(0-20)$ \\
\hline \multicolumn{4}{|l|}{ Method } \\
\hline Open & $36(63.2)$ & $22(88.0)$ & $14(43.8)$ \\
\hline Laparoscopic & $18(31.6)$ & $2(8.0)$ & $16(50.0)$ \\
\hline Laparoscopic assistance & $3(5.3)$ & $1(4.0)$ & $2(6.3)$ \\
\hline \multicolumn{4}{|l|}{ Primary anastomosis } \\
\hline Overall intention to treat & $53(93.0)$ & $22(88.0)$ & $31(96.9)$ \\
\hline Successful stenting ${ }^{\dagger}$ & $41 / 43(95.3)$ & $14 / 15(93.3)$ & $27 / 28(96.4)$ \\
\hline Unsuccessful stenting ${ }^{\dagger}$ & $12 / 14(85.7)$ & $8 / 10(80.0)$ & $4 / 4(100.0)$ \\
\hline \multicolumn{4}{|l|}{ Stoma creation } \\
\hline Overall intention to treat & $6(10.5)$ & $5(20.0)$ & $1(3.1)$ \\
\hline Successful stenting ${ }^{\dagger}$ & $2 / 43(4.7)$ & $1 / 15(6.7)$ & $1 / 28(3.6)$ \\
\hline Unsuccessful stenting $^{\dagger}$ & $4 / 14(28.6)$ & $4 / 10(40.0)$ & $0 / 4(0)$ \\
\hline \multicolumn{4}{|l|}{ Length of stay* (day) } \\
\hline Post stenting & $4 \pm 2(1-10)$ & $5 \pm 3(2-8)$ & $3 \pm 2(1-10)$ \\
\hline Post resection & $6 \pm 5(3-25)$ & $6 \pm 5(3-68)$ & $6 \pm 4(3-25)$ \\
\hline Total (stent and resection) & $11 \pm 12(5-76)$ & $11 \pm 13(3-76)$ & $10 \pm 11(5-32)$ \\
\hline
\end{tabular}

*Data presented as median \pm standard deviation (range). + Value of $\mathrm{n}$ is provided for patients who were included in analysis, and percentages are calculated according to this value. 

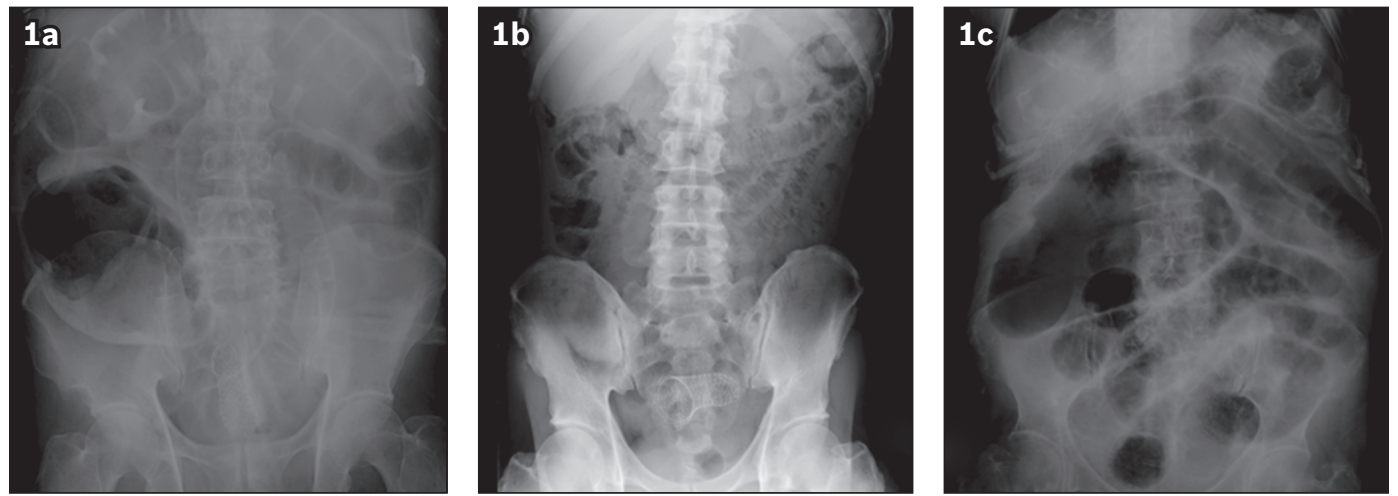

Fig. 1 (a) Radiograph shows persistent large bowel and caecal dilatation with pneumoperitoneum after stenting of an obstructing rectosigmoid tumour on Day 3 despite adequate decompression, with liquid stools seen after stent insertion. The patient had abdominal pain, was noted to have perforation at the caecum intraoperatively, and underwent an open right hemicolectomy and anterior resection. (b) Radiograph shows stent in situ but evidence of pneumoperitoneum (Rigler's sign) ten days after stenting of an obstructing sigmoid tumour with clinical success on discharge. The patient presented with abdominal pain and underwent a Hartmann's procedure, which found a 5-mm perforation just proximal to the stent. (c) Radiograph shows stent in situ but a distended caecum and small bowel 12 days after stenting of an obstructing transverse colon tumour with clinical evidence of decompression thereafter. The patient had worsening abdominal distension and underwent an open extended right hemicolectomy, during which the colonic stent was noted to be loose and able to freely slip in and out of the tumour.

Table IV. Final histopathology staging of resected specimens.

\begin{tabular}{|llll|}
\hline Stage & \multicolumn{3}{c|}{ No. (\%) } \\
\cline { 2 - 4 } & $\begin{array}{l}\text { Overall } \\
(\mathbf{n}=\mathbf{7 5})\end{array}$ & $\begin{array}{l}\mathbf{2 0 0 9 - 2 0 1 1} \\
(\mathbf{n}=\mathbf{3 6})\end{array}$ & $\begin{array}{l}\mathbf{2 0 1 2 - 2 0 1 5} \\
(\mathbf{n}=\mathbf{3 9})\end{array}$ \\
\hline I & $0(0)$ & $0(0)$ & $0(0)$ \\
\hline II & $15(20.0)$ & $6(16.7)$ & $9(23.1)$ \\
\hline III & $36(48.0)$ & $17(47.2)$ & $19(48.7)$ \\
\hline VI* & $24(32.0)$ & $13(36.1)$ & $11(28.2)$ \\
\hline
\end{tabular}

*Non-resected tumours with radiological evidence of metastasis were considered Stage VI.

emergency surgery, resulting in shorter hospital stays and lower postprocedural complication rates. In their study, clinical and technical success rates were high, and there was little difference between the BTS and palliation groups. The average technical success rate was $96.2 \%$ and clinical success rate was $92.0 \%$. Their results are comparable to those of the present series and suggest that stenting is a successful procedure with promising outcomes for the relief of malignant bowel obstruction and a low, acceptable rate of complications. Another pooled analysis by Sebastian et $\mathrm{al}^{(14)}$ had largely similar results to Watt et al's review, with $94 \%$ technical success and $91 \%$ clinical success. Clinical success was substantially higher in the palliative group. Stent migration was found to be the most common complication, occurring in $11.81 \%$ of included patients. The analysis also suggested that stents are a safe and effective alternative to emergency surgery.

SEMS has important advantages over emergency surgery. Not only can the patient undergo a significantly less invasive procedure (especially salient to palliative patients who can avoid surgery if possible), studies have shown that surgery is technically more successful after stenting. ${ }^{(14)}$ This is due to the ability to optimise the patient's comorbid state prior to the operation, including maximising their nutritional status, ensuring adequate bowel preparation and rectifying electrolyte disturbances. A BTS technique also makes it possible for laparoscopic oncological surgical resection to be performed after adequate bowel decompression, as shown in the second half of our study during

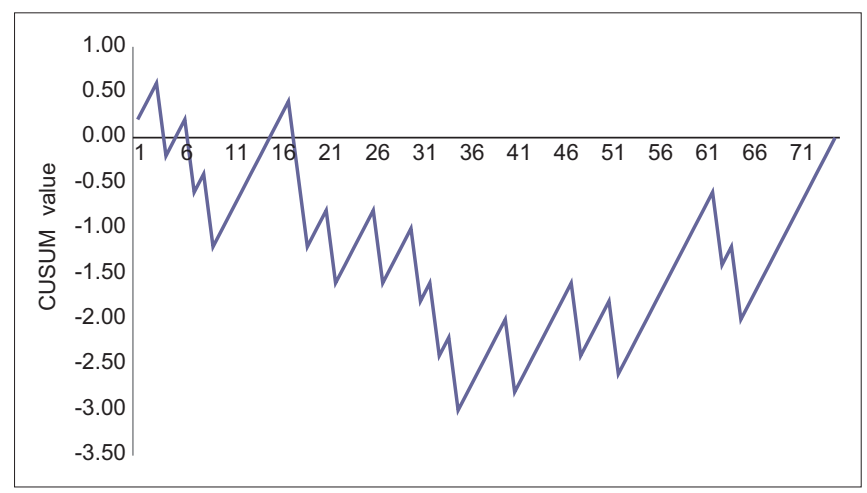

Fig. 2 Cumulative sum graph shows the cumulative clinical success rates (x-axis) plotted against consecutive cases of colonic stenting ( $y$-axis).

which the majority of patients had laparoscopic surgery. A metaanalysis by Zhang et al concluded that stenting, as opposed to emergency surgery, in a BTS population reduces intensive care length of stay, generates higher primary rates of anastomosis, and lowers both stoma and leak rates. ${ }^{(7)}$ A recent Cochrane review by Sagar of five randomised controlled trials found that SEMS reduces hospital stays and the duration of surgery, although it found no difference in overall mortality or morbidity when comparing stenting and surgery. ${ }^{(15)}$ The first randomised controlled trial from the University of Birmingham Clinical Trials Unit, comparing endoluminal stenting to emergency surgery, demonstrated in an early abstract publication that stenting as BTS decreases the rate of stoma formation without affecting overall mortality. ${ }^{(16)}$

Despite the short-term advantages of colonic stenting in terms of shorter hospital stay, higher anastomotic rates and lower stoma rates, almost all reviews have shown that the long-term survival outcomes are at best comparable to the traditional method, emergency surgical resection of obstructed colorectal cancer. While emergency surgery almost guarantees successful bowel decompression, the application of SEMS is associated with both technical and clinical failure, which leads to inevitable emergency surgery. The associated risk of stent perforation is approximately 
$4 \%{ }^{(15)}$ Whether this affects subsequent tumour recurrence is an area of great debate. Although SEMS is beneficial to patients when it is successfully deployed with bowel decompression, the success rates are dependent on many factors.

It is important to identify the patients who are suitable for stenting based on characteristics such as patient factors and tumour location. Van Hooft et al have suggested that the subset of patients suitable for stenting should be defined further. ${ }^{(12)}$ Blake et al carried out a United Kingdom-based case series on 54 patients that had lower success rates than in other studies. ${ }^{(9)}$ Technical success was $86 \%$ and clinical success was $84 \%$, while complications occurred in one-fifth of patients. We may speculate that clinician experience was more limited at this centre given its status as a district general hospital, therefore accounting for its lower success rates than in other publications.

Technical failures are mostly due to the inability to locate the lumen so that the guidewire can be safely passed through. In three out of four cases in the present study, the tumour was at the curved portion of the colon. While this is not an absolute contraindication to stenting, it is important to note that there is a higher failure rate in such cases.

The main reasons for the clinical failures in our study were inadequate decompression (seven cases) and perforation (two cases). The size of the stent plays an important role in the clinical success of SEM. Prior to 2012, the majority of stents used were those with the larger 25-mm diameter. In addition to concern about a higher risk of perforation with the larger stents, we have also noted a significant amount of intraoperative fibrosis, which makes resection more difficult. As such, we have more recently moved towards using the smaller 22-mm stents in selected patients. Although there were cases of inadequate decompression due to very hard impacted stools proximally in our study cohort, there was no overall discernible difference in clinical success rates between the two stent sizes.

Another crucial factor is the experience and skill level of the endoscopist. This is commonly highlighted in discussions on the factors involved in stent failure but is a difficult area to further investigate. The failure rates of pooled meta-analysis data are usually lower than those in single-operator case series, possibly reflecting the importance of the caseload and experience of the endoscopist in the outcome of colonic stenting. ${ }^{(8-10)}$ Our study showed an improvement in success rates in the second half, after the endoscopist gained more experience with colonic stenting. The success rates were also superior to results of a previous randomised study that was conducted locally in the same institution. ${ }^{(17)}$ Our CUSUM graph showed that the clinical success rate improved only after performing 35 cases of colonic stenting. In order to build up experience in endoscopic colonic stenting, our institution has designated endoscopists who receive referrals of suitable cases primarily for stenting, while the subsequent resections can be performed by other colorectal surgeons.

Most of the resistance to SEMS as a BTS is due to concern regarding its higher disease recurrence rates, and therefore lower survival rates, as a result of perforation from colonic stenting. However, Sagar showed that there are no differences in long-term outcomes and survival rates between those who have stenting followed by resection and those undergoing emergency bowel resection. ${ }^{(15)}$

Colonic stenting remains an attractive alternative to emergency surgery for malignant colonic obstruction, especially for patients with high operative morbidity and mortality. It allows definitive surgical resection to be performed in an elective setting, with the option of laparoscopic surgery and potentially avoiding a stoma. While stenting for proximal lesions is considered technically more challenging and not usually recommended, we have shown that proximal stenting is feasible and can be considered in suitable patients. A large cohort study on stenting for proximal lesions also showed very encouraging results. ${ }^{(18)}$

Our study had some limitations. First, although a single surgeon placed the stents in all patients, different surgeons performed the subsequent elective surgery. We did not examine the effect of this on our outcomes. Additionally, as with all retrospective studies projects, it is possible that a degree of selection bias may have been present.

In conclusion, using SEMS appears to be a safe and effective way of relieving large bowel obstruction caused by colorectal cancer, even for proximal lesions. The complication rate found in our series was low and success rates were on par with those in the existing literature. After successful stent placement, patients were subsequently able to undergo surgical resection in an elective setting with minimal complications. We have also shown that the stoma rate is significantly lower in cases where stenting was successful and that the success rate correlates with experience. It is therefore imperative that colonic stenting is performed by an experienced endoscopist, in terms of technical skill and knowledge of patient selection, due to the learning curve required. This would generate a higher success rate with fewer complications. We hope to see more high-quality studies in this area.

\section{REFERENCES}

1. Colorectal cancer statistics. In: World Cancer Research Fund International [online]. Available at: http://www.wcrf.org/int/cancer-facts-figures/data-specificcancers/colorectal-cancer-statistics\#BOTH. Accessed September 1, 2015.

2. Cirocchi R, Farinella E, Trastulli S, et al. Safety and efficacy of endoscopic colonic stenting as a bridge to surgery in the management of intestinal obstruction due to left colon and rectal cancer: a systematic review and meta-analysis. Surg Oncol 2013; 22:14-21.

3. Sjödahl R, Franzén T, Nyström PO. Primary versus staged resection for acute obstructing colorectal carcinoma. Br J Surg 1992; 79:685-8.

4. Tekkis PP, Poloniecki JD, Thompson MR, Stamatakis JD. Operative mortality in colorectal cancer: prospective national study. BMJ 2003; 22;327:1196-201.

5. Nugent KP, Daniels P, Stewart B, Patankar R, Johnson CD. Quality of life in stoma patients. Dis Colon Rectum 1999; 42:1569-74.

6. Dohmoto M. New method-endoscopic implantation of rectal stent in palliative treatment of malignant stenosis. Endosc Digest 1991; 3:1507-12.

7. Zhang Y, Shi J, Shi B, et al. Self-expanding metallic stent as a bridge to surgery versus emergency surgery for obstructive colorectal cancer: a meta-analysis. Surg Endosc 2012; 26:110-9.

8. Tan CJ, Dasari BV, Gardiner K. Systematic review and meta-analysis of randomized clinical trials of self-expanding metallic stents as a bridge to surgery versus emergency surgery for malignant left-sided large bowel obstruction. Br J Surg 2012; 99:469-76.

9. Blake P, Delicata R, Cross N, Sturgeon G, Hargest R. Large bowel obstruction due to colorectal carcinoma can be safely treated by colonic stent insertion--case series from a UK district general hospital. Colorectal Dis 2012; 12:1489-92.

10. Mehmood RK, Parker J, Kirkbride P, et al. Outcomes after stenting for malignant large bowel obstruction without radiologist support. World J Gastroenterol 2014; 20:6309-13. 
11. van Hooft JE, Fockens P, Marinelli AW, et al; Dutch Colorectal Stent Group. Early closure of a multicentre randomized clinical trial of endoscopic stenting versus surgery for stage IV left-sided colorectal cancer. Endoscopy 2008; 40:184-91.

12. van Hooft JE, Bemelman WA, Oldenburg B, et al; collaborative Dutch Stent-In study group. Colonic stenting versus emergency surgery for acute left-sided malignant colonic obstruction: a multicentre randomised trial. Lancet Oncol $2011 ; 12: 344-52$.

13. Watt AM, Faragher IG, Griffin TT, Rieger NA, Maddern GJ. Self-expanding metallic stents for relieving malignant colorectal obstruction: a systematic review. Ann Surg 2007; 246:24-30.

14. Sebastian S, Johnston S, Geoghegan T, Torreggiani W, Buckley M. Pooled analysis of the efficacy and safety of self-expanding metal stenting in malignant colorectal obstruction. Am J Gastroenterol 2004; 99:2051-7.

15. Sagar J. Colorectal stents for the management of malignant colonic obstructions. Cochrane Database Syst Rev 2011; 9:CD007378.

16. Hill J, Kay C, Morton D, et al. CREST: randomised phase III study of stenting as a bridge to surgery in obstructing colorectal cancer--results of the UK ColoRectal Endoscopic Stenting Trial (CREST). J Clin Oncol 2016; 15 Suppl:3507.

17. Ho KS, Quah HM, Lim JF, Tang CL, Eu KW. Endoscopic stenting and elective surgery versus emergency surgery for left-sided malignant colonic obstruction: a prospective randomized trial. Int J Colorectal Dis 2012; 27:355-62.

18. Yao LQ, Zhong YS, Xu MD, et al. Self-expanding metallic stents drainage for acute proximal colon obstruction. World J Gastroenterol 2011; 17:3342-6. 